\title{
Enabling Energy-Aware Mobile Data Offloading for Smartphones through Vertical Collaboration
}

\author{
Aaron Yi Ding*, Pan Hui ${ }^{\dagger}$, Markku Kojo*, and Sasu Tarkoma* \\ *Department of Computer Science, University of Helsinki, P.O. Box 68, FI-00014, Finland \\ ${ }^{\dagger}$ Deutsche Telekom Laboratories, Ernst-Reuter-Platz 7, 10587 Berlin, Germany
}

\begin{abstract}
Searching for mobile data offloading solutions has become topical in recent years. In the Metropolitan Advanced Delivery Network (MADNet) project, we explore the potential of multiple interfaces on smartphones through a collaborative design to enable energy-aware WiFi-based mobile data offloading. We advocate that the vertical collaboration across different wireless access technologies and network vendors is the key to aggregate the power of mobile operators, $\mathrm{WiFi}$ service providers, and end-users. Aiming at reducing the energy consumption on smartphones, we propose an energyaware algorithm supported by our collaborative architecture to avoid offloading to low-throughput WiFi networks which may consume more energy. The evaluation of streaming applications on our prototyped Nokia N900 smartphones demonstrates that we are able to achieve more than $80 \%$ energy saving. Our experiment in the wild also shows that our design can tolerate the minor errors of localization, mobility prediction, and offload capacity estimation.
\end{abstract}

\section{Categories and Subject Descriptors}

C.2.1 [Network Architecture and Design]: Network communications

\section{Keywords}

Energy aware, vertical collaboration, mobile data offloading

\section{INTRODUCTION}

Mobile data networks are currently overloaded by various bandwidth-hungry smartphone applications such as HD mobile TV, especially in metropolitan areas [4]. Although mobile operators are upgrading their infrastructure to LTE (4G) with higher capacity to meet such growing demand, the pace of infrastructure upgrade is still behind the radical growth of user generated data traffic. This imbalance between supply and demand has led to severe deterioration of service quality because mobile networks can not cope with

Permission to make digital or hard copies of all or part of this work for personal or classroom use is granted without fee provided that copies are not made or distributed for profit or commercial advantage and that copies bear this notice and the full citation on the first page. To copy otherwise, to republish, to post on servers or to redistribute to lists, requires prior specific permission and/or a fee.

CoNEXT Student'12, December 10, 2012, Nice, France.

Copyright 2012 ACM 978-1-4503-1779-5/12/12 ...\$15.00. the sudden growth [1]. To relieve this problem, mobile data offloading is sought as a promising direction by using complementary communication technologies such as WiFi to deliver traffic originally planned for mobile data networks.

Owing to the maturity of WiFi technology and its vast deployment, WiFi-based mobile data offloading has been shown to be feasible $[6,1,5]$. However, there are still several challenging issues when offloading mobile data traffic for smartphones. First, most of existing proposals such as Wiffler and oSCTP $[1,5]$ are designed for PCs on vehicular network without considering the offloading energy consumption and difference of antenna deployed on mobile devices. Such problem has been explored and discussed in recent analysis [7]. Lee et al. [6] evaluate the energy saving through a trace-driven simulation with simplified assumptions, but how to harvest the energy gain of mobile data offloading in practice is still an open problem. Second, through extensive measurement using smartphones in four cities, we found that the number of open-accessible WiFi access points (APs) is very limited [3]. Therefore the schemes that utilize only open APs such as Wiffler [1] may not be enough. Finally, the goal of previous work is mainly to increase the amount of delay-tolerant traffic offloaded to WiFi networks, but delaytolerant applications generate only a small amount of data traffic comparing to streaming applications $[8,2]$.

To address these challenges, we propose the MADNet offloading architecture by exploiting the vertical collaboration across mobile networks, WiFi accesses, and end-users to achieve energy-aware mobile data offloading for smartphones.

\section{DESIGN \& ALGORITHM}

The core of MADNet includes three software components: a client module on smartphones (Smart-Client), a proxy on WiFi APs (Proxy-WiFi), and another proxy on a cellular access network (Proxy-Cellular). The offloading process involves collaboration among all three components following the design principle of vertical collaboration. Each component contributes necessary context and available resources to enable energy-aware mobile data offloading.

To make an offloading decision, MADNet utilizes the contextual information from network vendors and smartphones. First, Proxy-Cellular knows the location of neighboring WiFi APs of a given smartphone since these locations are fixed, deployed by mobile operators, or obtainable through WiFi providers and services such as wigle.net. Second, as people are creatures of habit, it is feasible to predict their mobility patterns using history information [9]. The energy-aware 


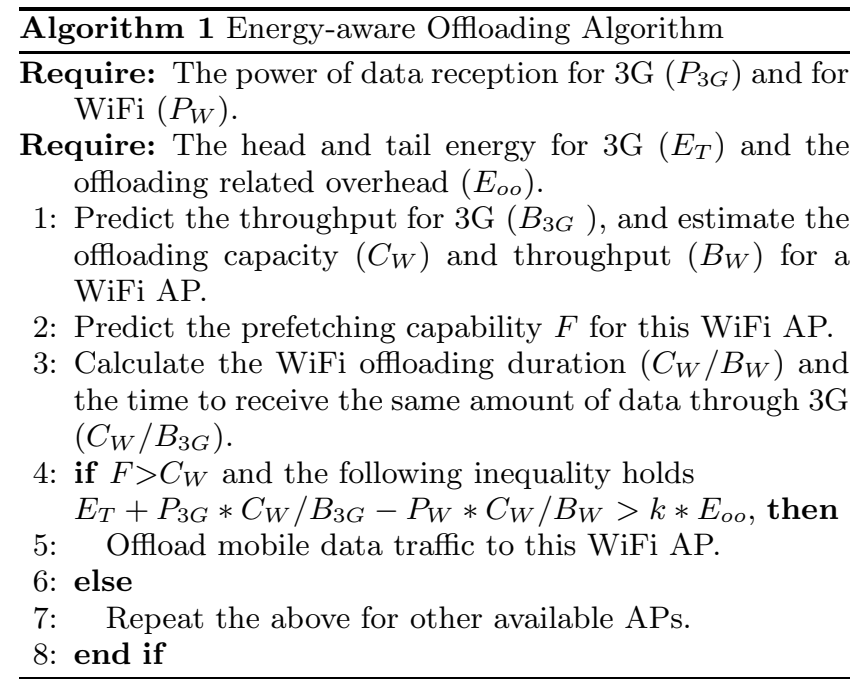

offloading is enabled by our simple yet efficient algorithm running on Proxy-Cellular, as illustrated in Algorithm 1.

To demonstrate MADNet in a practical scenario, we first explore an energy efficient solution to offload real-time streaming traffic for smartphones that are in motion. Since when people walk or ride on bikes, although they do not read news or watch videos, they often listen to music. We thus implement our proposal on three major components accordingly.

\section{PERFORMANCE EVALUATION}

We first focus on the case that each Proxy-WiFi will serve one Smart-Client at a time and thus guarantee the offloading performance. We note that we can extend the MADNet architecture to support multiple devices simultaneously by integrating efficient scheduling algorithms.

We evaluate the effectiveness of our design by comparing the amount of prefetched data and actual volume downloaded by Smart-Client through our walking tests of 20 runs in Helsinki ( $k$ set to 1.1), as shown in Figure 1. According to the results we can sufficiently utilize the WiFi link for $85 \%$ of runs as the amount of downloading is smaller than that of prefetching and the average gap is around 4.8 MB. The results also demonstrate that our proposal can tolerate the minor errors resulting from WiFi positioning, mobility prediction, and offload capacity estimation in a live environment. Based on our energy measurements [3], we present the estimated energy consumption of $3 \mathrm{G}$ and $\mathrm{WiFi}\left(E_{3 G}, E_{W}\right)$ and the energy saving for our tests in Table 1 . These results show that MADNet can save more than $80 \%$ of energy consumption on smartphones benefiting from the collaborative architecture.

\section{SUMMARY}

We present the MADNet architecture harvesting the vertical collaboration among mobile operators, WiFi providers and end-users to improve energy saving for smartphones. We confirm the feasibility of our proposal through a prototype implementation and evaluate its performance in the wild, which further verifies the effectiveness of our scheme. Currently we are extending the collaborative design for multiple mobile devices and working with a cellular operator for a possible future deployment.

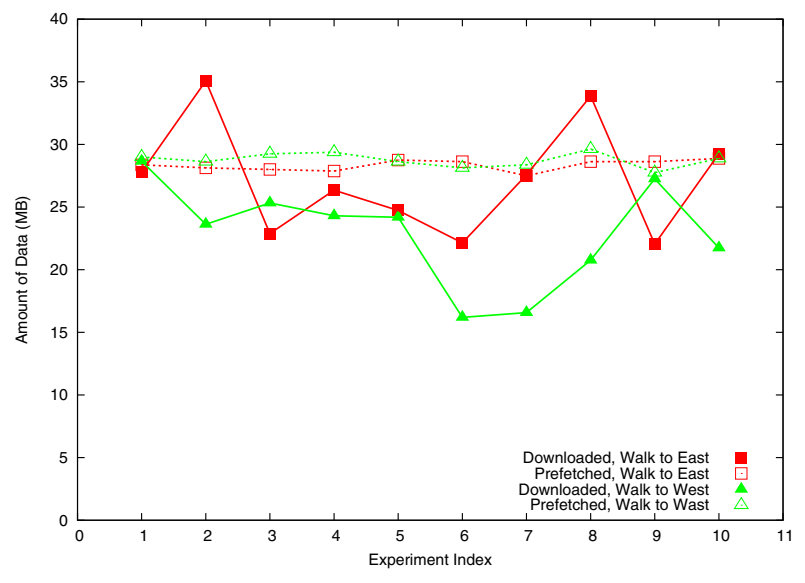

Figure 1: Offloading Performance

Table 1: Measurement Results and Energy Saving

\begin{tabular}{|c|c|c|c|c|c|c|c|}
\hline & $C_{W}$ & $B_{3 G}$ & $B_{W}$ & $E_{o o}$ & $E_{3 G}$ & $E_{W}$ & Saving \\
\cline { 2 - 8 } & $\mathrm{MB}$ & $\mathrm{Mbps}$ & $\mathrm{Mbps}$ & Joule & Joule & Joule & $\%$ \\
\hline East & 27.2 & 0.8 & 3.5 & 3.4 & 292.8 & 39.3 & 85.42 \\
West & 22.9 & 0.8 & 3.2 & 3.5 & 256.4 & 38.9 & 83.46 \\
\hline
\end{tabular}

\section{Acknowledgments}

Authors would like to thank Dr. Bo Han from AT\&T Labs for his support and guidance on this work. The $\mathrm{PhD}$ research of Aaron Yi Ding is supported by the Nokia Foundation and the Academy of Finland.

\section{REFERENCES}

[1] A. Balasubramanian et al. Augmenting Mobile 3G Using WiFi. In Proceedings of ACM MobiSys 2010.

[2] H. Falaki et al. A First Look at Traffic on Smartphones. In Proceedings of ACM IMC 2010.

[3] B. Han et al. Energy-Aware Collaborative Mobile Data Offloading for Smartphones. Technical Report, University of Maryland. Available at http://www.cs.umd.edu/ bohan/MADNet.pdf.

[4] B. Han et al. Mobile Data Offloading through Opportunistic Communications and Social Participation. IEEE Transactions on Mobile Computing, 11(5):821-834, 2012.

[5] X. Hou et al. Moving Bits from 3G to Metro-Scale WiFi for Vehicular Network Access: An Integrated Transport Layer Solution. In Proceedings of IEEE ICNP 2011.

[6] K. Lee et al. Mobile Data Offloading: How Much Can WiFi Deliver? In Proceedings of ACM CoNEXT 2010.

[7] S. Liu and A. Striegel. Casting Doubts on the Viability of WiFi Offloading. In Proceedings of ACM CellNet Workshop at SIGCOMM 2012.

[8] G. Maier, F. Schneider, and A. Feldmann. A First Look at Mobile Hand-held Device Traffic. In Proceedings of PAM 2010.

[9] A. Nicholson and B. Noble. BreadCrumbs: Forecasting Mobile Connectivity. In Proceedings of ACM MobiCom 2008, September 2008. 